\title{
Integrated genomic analysis identifies clinically relevant subtypes of renal clear cell carcinoma
}

Peng Wu ${ }^{1,2 \dagger}$, Jia-Li Liu ${ }^{3 \dagger}$, Shi-Mei Pei ${ }^{2,4 \dagger}$, Chang-Peng Wu ${ }^{3}$, Kai Yang ${ }^{1,2}$, Shu-Peng Wang ${ }^{1,2}$ and Song Wu ${ }^{1,2^{*}}$

\begin{abstract}
Background: Renal cell carcinoma (RCC) account for over $80 \%$ of renal malignancies. The most common type of RCC can be classified into three subtypes including clear cell, papillary and chromophobe. cCRCC (the Clear Cell Renal Cell Carcinoma) is the most frequent form and shows variations in genetics and behavior. To improve accuracy and personalized care and increase the cure rate of cancer, molecular typing for individuals is necessary.

Methods: We adopted the genome, transcriptome and methylation HMK450 data of ccRCC in The Cancer Genome Atlas Network in this research. Consensus Clustering algorithm was used to cluster the expression data and three subtypes were found. To further validate our results, we analyzed an independent data set and arrived at a consistent conclusion. Next, we characterized the subtype by unifying genomic and clinical dimensions of ccRCC molecular stratification. We also implemented GSEA between the malignant subtype and the other subtypes to explore latent pathway varieties and WGCNA to discover intratumoral gene interaction network. Moreover, the epigenetic state changes between subgroups on methylation data are discovered and Kaplan-Meier survival analysis was performed to delve the relation between specific genes and prognosis.
\end{abstract}

Results: We found a subtype of poor prognosis in clear cell renal cell carcinoma, which is abnormally upregulated in focal adhesions and cytoskeleton related pathways, and the expression of core genes in the pathways are negatively correlated with patient outcomes.

Conclusions: Our work of classification schema could provide an applicable framework of molecular typing to ccRCC patients which has implications to influence treatment decisions, judge biological mechanisms involved in ccRCC tumor progression, and potential future drug discovery.

Keywords: $\mathrm{ccRCC}$, Gene expression, Molecular classification, Pathway

\section{Background}

ccRCC, the most common type of kidney cancer, representing approximately $92 \%$ of such cases. Most people with kidney cancer are usually over 55 years of ages and this cancer is more common in men [1].The global pattern of genetic changes underlying ccRCC includes alterations in genes controlling cellular oxygen sensing and the maintenance of chromatin states [2]. Early mutations

\footnotetext{
* Correspondence: wusong@szu.edu.cn

${ }^{\dagger}$ Equal contributors

${ }^{1}$ The Affiliated Luohu Hospital of Shenzhen University, Department of Urological Surgery, Shenzhen University, Shenzhen 518000, China

${ }^{2}$ Shenzhen Following Precision Medical Institute, Shenzhen Luohu Hospital

Group, Shenzhen 518000, China

Full list of author information is available at the end of the article
}

and inactivation of VHL is commonly seen in ccRCC [3]. Other recurrently mutated genes include PBRM1, BAP1 and SETD2, located in chromosome 3p, whose loss is the most frequent arm-level events inccRCC $(91 \%$ of samples) [4]. Losses on chromosome 14q and gains of $5 \mathrm{q}$ were also frequent observed, specially, the former is associated with more aggressive phenotype [5]. These genetic aberrations are critical for clinical diagnosis and personal therapy. We collected gene expression data of ccRCC from TCGA and using Consensus Clustering [6] algorithm cluster all samples to detect potential subtypes. We discovered three subtypes and survival analysis showed one subclass has far poorer prognosis than the other three. Thus, we compared the poor subclass 
with the other and find some pathways changes and genes that may cause adverse outcomes.

\section{Methods}

\section{Consensus clustering identified three subtypes of ccRCC}

Features of Consensus Clustering algorithm are the 2D feature and item subsampling and it provides a method to represent the consensus across multiple runs of a clustering algorithm, to determine the number of clusters in the data, and to assess the stability of the discovered clusters [6]. The method can also be used to represent the consensus over multiple runs of a clustering algorithm with random restart (such as K-means, model-based Bayesian clustering, SOM, etc.), so as to account for its sensitivity to the initial conditions [7].This method has gained popularity in cancer genomics, where new molecular subclasses of disease have been discovered [8-11].

We select the samples of ccRCC in expression data which contain molecular subtypes in TCGA and filter out samples without molecular information and genes with low signal across samples to get more precise classification results. We classify samples into three robust expression clusters (EC) utilizing Consensus Clustering together with hierarchical clustering. The clustering stability increases from $\mathrm{k}=2$ to $\mathrm{k}=3$, but not for $\mathrm{k}>3$ (Fig. 1a and b) and delta area under the curve in $k=3$ also has appreciable increase (Fig. 1c). Combined with the clinical data, we performed Kaplan meier analysis and the survival curve shows the EC1 subtype are obviously more malignant than the other (Fig. 2a).

\section{Validation of subtypes in an independent data set}

To validate our results, an independent data set including 265 ccRCC patients from GEO was used to assess the subtype reproducibility [12]. We visualize the expression data by a 164 classifying marker gene list and hierarchical clustering. The marker genes were identified in EC1-3 subtypes by combining Wilcoxon signed-rank test and permutation (see methods). Unsurprisingly, the validation data set almost coincided with the data set of a

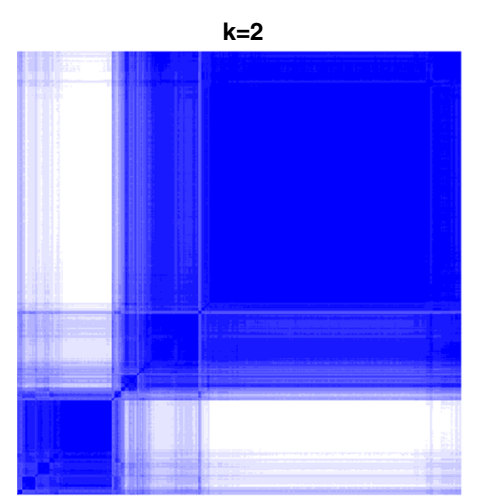

$k=4$

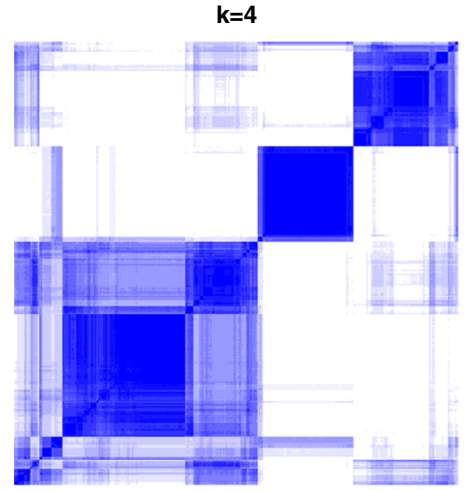

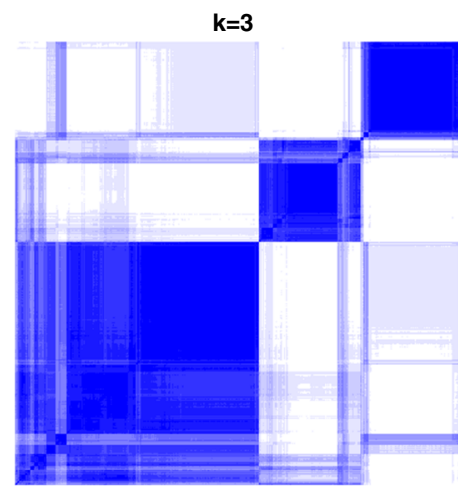

$k=5$

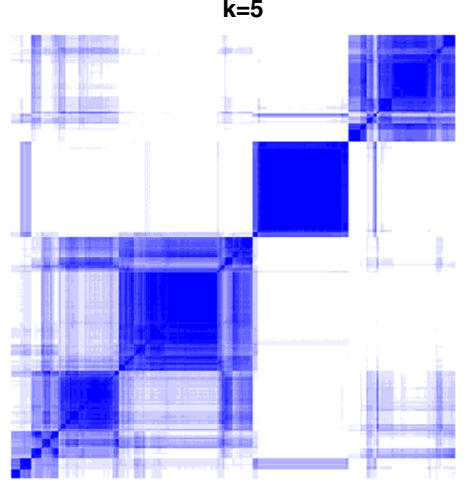

b

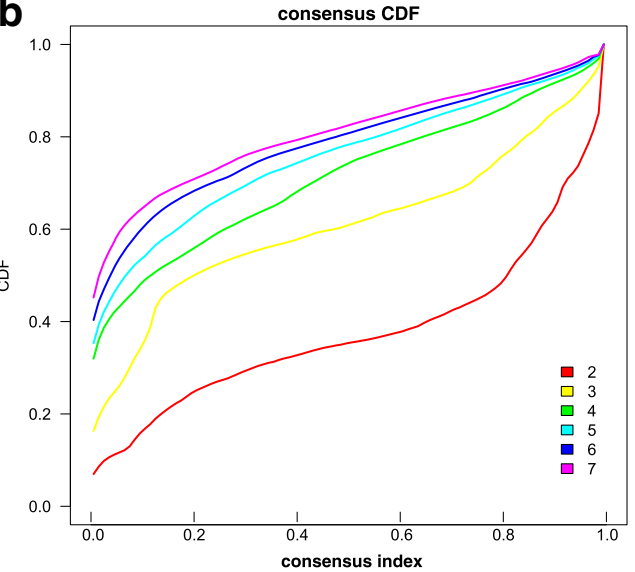

C

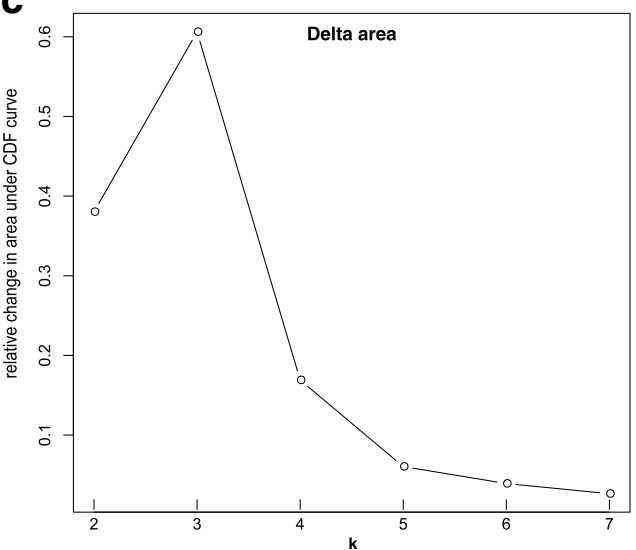

Fig. 1 a Consensus matrices. Both rows and columns represent samples and consensus values range from 0(never clustered together) to 1 (always clustered together) marked by white to dark blue. b Consensus Cumulative Distribution Function (CDF) Plot. CDF plot shows the cumulative distribution functions of the consensus matrix for each $\mathrm{k}$ (indicated by colors) c Delta Area Plot. This graphic shows the relative change in area under the CDF curve. In $k=3$, the shape of the curve approaches the ideal step function, and shape hardly changes as we increase $K$ past 3 


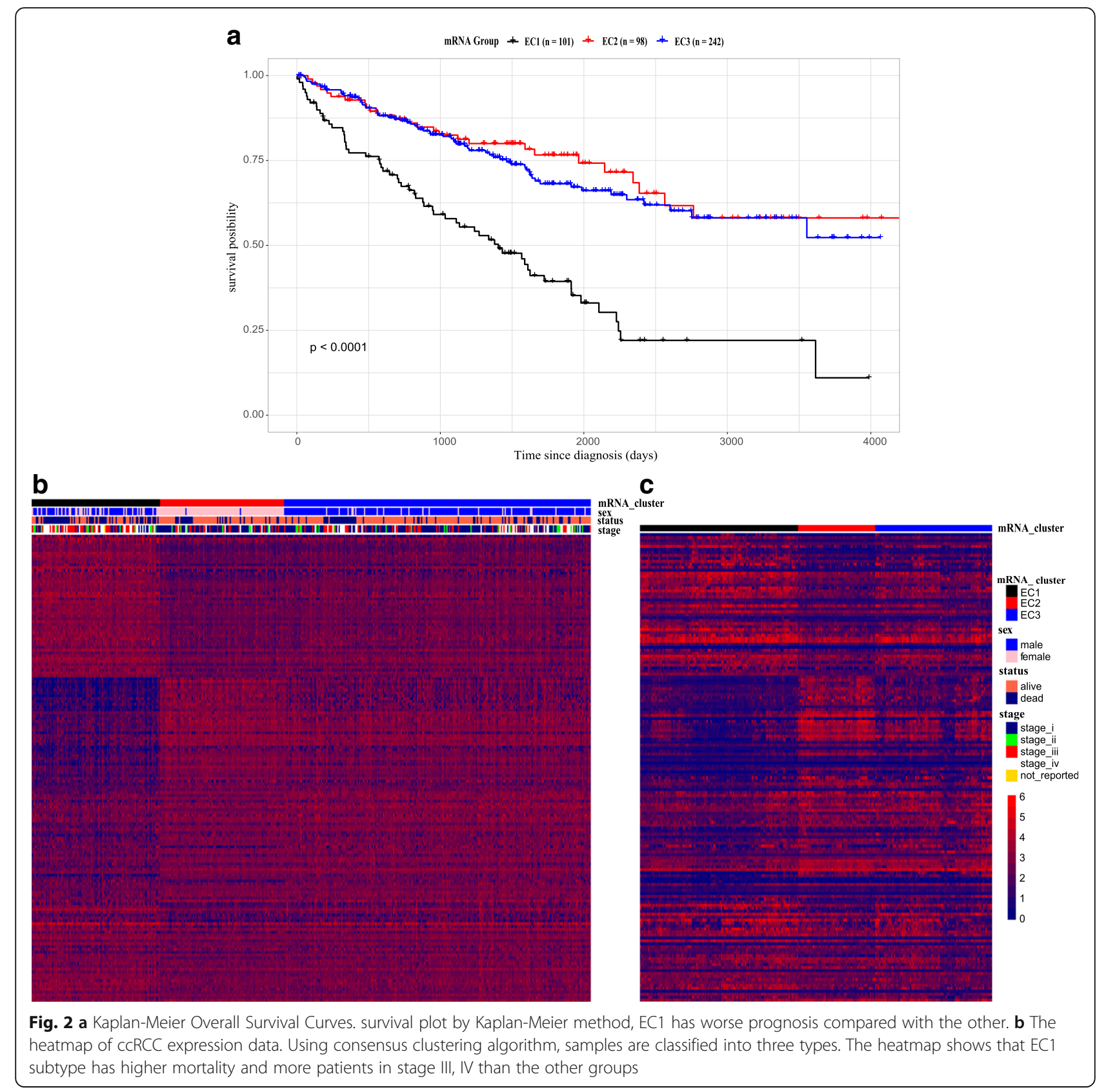

TCGA, comprising of three subtypes (Additional file 1: Figure S1) and representing similar expression profile (Fig. $2 \mathrm{~b}$ and Fig. 2c). Considering differences in sample size and different sequencing techniques, obvious concordance was seen between our classification and the results from the earlier study, which further proves the reliability of our analysis and the authenticity of the three subtypes.

\section{Genetic aberrations and Clinicopathological parameters of subtypes}

We classified the three types into malignant and relative unmalignant types because of survival analysis results which shows EC2-3 has approximate prognosis. We summarized the copy number mutations and single nucleotide variation of $\mathrm{EC} 1$ and top five recurrent mutation genes are VHL, PBRM1, MUC4, BAP1 and SETD2 $(42.57 \%, 25.74 \%, 20.79 \%, 19.80 \%, 13.86 \%)$. Frequency of frequently mutated genes except BAP1 were similar between these two types (Table 1), but the high-level somatic copy number variation (SCNV) regions between two groups were quite different (Table 2). BAP1, a nuclear deubiquitinase, is inactivated in $15 \%$ of ccRCCs. A significant increase in BAP1 mutation frequency was observed in EC1compared with the remainder of the 
Table 1 Mutation frequency of genes with single nucleotide variations in two groups

\begin{tabular}{lllll}
\hline Genes & EC1 & EC2-3 & $P$ values & All \\
\hline VHL & $42.57 \%$ & $54.41 \%$ & 0.0480 & $51.70 \%$ \\
PBRM1 & $25.74 \%$ & $35.35 \%$ & 0.0948 & $33.10 \%$ \\
MUC4 & $20.79 \%$ & $18.25 \%$ & 0.3941 & $18.82 \%$ \\
BAP1 & $19.80 \%$ & $5.89 \%$ & $4.508 \mathrm{e}-05$ & $9.07 \%$ \\
SETD2 & $13.86 \%$ & $12.93 \%$ & 0.9421 & $13.15 \%$ \\
\hline
\end{tabular}

samples, which is consistent with BAP1 is a potential tumor suppressor and relevant to bad outcome in ccRCC $[13,14]$.

Gain of $5 q$ paired with loss of $3 p$ was observed frequent in both EC1 and EC2-3 when it is a highly frequent event in ccRCC. However, loss of 9q21 presents a higher frequency in EC1 and three common tumor suppressor genes (TSG) are deleted in this area including CDKN2A, CDKN2B and MTAP. CDKN2A and CDKN2B act as tumor suppressors by regulating the cell cycle which block traversal from G1 to S-phase or inhibits cell cycle G1 progression. The deletion, mutation or promoter methylation of the two genes are common in various cancers, which help to the unlimited growth of cancer cells and CDKN2A is associated with metastatic cancer [15-18]. MTAP is key enzyme in the methionine salvage pathway and frequently deleted in human cancers because of its chromosomal proximity to CDKN2A [19]. This SCNA pattern may conduce to increase the potential of proliferation for EC1 subtype.

The clinical and pathological features are largely distinct from each other (Table 3). We compare EC1 with EC2-3 in the two datasets from four perspectives: age, gender, grade and stage and estimate the significance by chi-squared test of $2 * 2$ table. Besides gender, EC1 is highly interrelated with older age, advanced grade and stage in TCGA data, which partly explains the result that this subtype has a poor prognosis. In GEO data, the results are similar although the Pvalues are not statistically significant enough, probably due to the unavailable patient information. We will discuss these results detailly in the following analysis.

\section{Enrichment analysis reveals high potential of EC1 in proliferation and metastasis}

In order to reveal the statistically significant, concordant differences between EC1 and other subtypes, gene sets enrichment analysis (GSEA) algorithm (see methods) is used and we chose KEGG gene sets as predefined gene sets [20]. Consequently, 7 gene sets are upregulated in $\mathrm{EC} 1$ and 5 gene sets are upregulated in EC2-3. The 7 gene sets of EC1 mainly focus on cell proliferation and mobility containing Focal Adhesion, Regulation of Actin Cytoskeleton and Chemokine (Fig. 3a). These pathways implicate epithelial-to-mesenchyme transition (EMT), cell proliferation and migration, closely related to tumor progression and metastasis. By contrast, up-regulated pathways in EC2-3 are mainly involved in metabolism including PPAR signaling pathway and Cytochrome P450. Next, our analysis concentrates on the core enrichment genes, which contributes to the leading-edge subset within the predefined gene set and have high expression level, in up-regulated pathways of EC1 for its poor prognosis (see methods).

Actin Cytoskeleton pathway is significantly enriched and there are 12 genes among core enrichment (Additional file 2: Table S1). MAPK Signal pathway is enriched with 15 genes, but only FLNC is core enrichment gene (Additional file 3: Table S2). It is noteworthy that FLNC is also core enrichment gene in Focal Adhesion pathway (Additional file 4: Table S3) and it has been reported that FLNC can be a potential progression marker for the development of hepatocellular carcinoma [21]. LAMB3 is core enrichment gene in Focal Adhesion and Pathways in Cancer (Additional file 5: Table S4) and research shows that repressing LAMB3 inhibit mutant KRAS-Driven tumor growth [22]. LAMB3 is also associated with EMT, a crucial change that happens to cancer cells before metastasis, and several researches conclude that high expression of LAMB3 is correlated with tumor metastasis including oral squamous cell carcinoma, bladder cancer and breast cancer [23-25]. Other core enrichment genes in Pathways in Cancer including MMP9 and MMP2, together with LAMB3, which are components of the extracellular matrix, may be considered as a

Table 2 Known cancer genes in the high-level copy number variation regions

\begin{tabular}{|c|c|c|c|c|}
\hline & EC1 & Known cancer related genes in Region & EC2-3 & Known cancer related genes in Region \\
\hline \multicolumn{5}{|c|}{ High-level amplified events } \\
\hline \multirow[t]{3}{*}{ Cytoband } & $5 q 35$ & FGFR4/DOCK2 (9.09\%) & $5 q 35$ & FGFR4/DOCK2 (19.94\%) \\
\hline & $5 q 32$ & CD74/CSF1R (9.09\%) & $5 q 31$ & CTNNA1/NR3C1 (17.86\%) \\
\hline & $5 q 33$ & PDGFRB/ZNF300 (9.09\%) & $5 q 33$ & PDGFRB/ZNF300 (17.86\%) \\
\hline \multicolumn{5}{|c|}{ High-level deletion events } \\
\hline \multirow[t]{3}{*}{ Cytoband } & $9 \mathrm{p} 21$ & CDKN2A/CDKN2B (11.11\%) & $3 p 25$ & PPARG/RAF1NHL (15.18\%) \\
\hline & $9 p 23$ & PTPRD (7.07\%) & $3 p 21$ & PBRM1/SETD2/BAP1 (15.18\%) \\
\hline & $3 p 25$ & PPARG/RAF1NHL (6.06\%) & $3 p 22$ & TGFBR2/MYD88 (14.58\%) \\
\hline
\end{tabular}


Table 3 Clinical characteristics of subtypes

\begin{tabular}{|c|c|c|c|c|c|c|c|}
\hline & & \multicolumn{3}{|l|}{ TCGA data } & \multicolumn{3}{|l|}{ GEO data } \\
\hline & & EC1 & EC2-3 & $P$ values & $\mathrm{EC1}$ & $E C 2-3$ & $P$ values \\
\hline Age $($ mean $\pm S D)$ & & $63.5 \pm 10.9$ & $60.2 \pm 12.5$ & 0.01168 & NA & NA & NA \\
\hline \multirow[t]{2}{*}{ Gender } & male & 66 & 224 & 1 & 81 & 79 & 0.04636 \\
\hline & female & 35 & 116 & & 38 & 64 (3NA) & \\
\hline \multirow[t]{2}{*}{ Pathological grade } & Grade $1+2$ & 20 & 174 & 4.67e-08 & 43 & 69 & 0.05186 \\
\hline & Grade $3+4$ & 81 & 166 & & $74(2 \mathrm{NA})$ & 70 (7NA) & \\
\hline \multirow[t]{2}{*}{ Stage } & Stage I-II & 35 & 220 & $1.478 \mathrm{e}-07$ & 22 & 31 & 0.09323 \\
\hline & Stage III-IV & 66 & 120 & & 42 (55NA) & $30(85 \mathrm{NA})$ & \\
\hline
\end{tabular}

molecular biomarker for ccRCC progress and metastasis. Chemokine Signaling Pathway containing CCL5, CXCL9 and CCR5 are also upregulated in EC1 (Additional file 6: Table S5). Chemokines play an important role in tumor growth and angiogenesis, on the one hand, providing cytokines to promote tumor growth, on the other hand, improving matrix metalloproteinase activity, promoting tumor cell through the cell membrane so as to increase the probability of tumor metastasis. These features reveal that EC1 subtype are more invasive and has a higher likelihood of migration, indicating that patients with EC1 expression pattern are prone to distant metastasis, resulting in poor prognosis. To evaluate the proliferation ability of subtypes, we next scored each sample for the expression signatures for the G1/S and $G 2 / M$ phases. EC1 has higher expression level than EC2-3 (C1/S: Ttest Pvalue = 9.486514e-07, G2/M: Ttest Pvalue =1.371606e-06), which reflects, to some extent, that the subtype has a stronger proliferative capacity (Fig. 3b). Moreover, we perform differential genes analysis between EC1 and EC2-3 to evaluate the significance of expression difference and find 11 genes that upregulated in 4 pathways of EC1
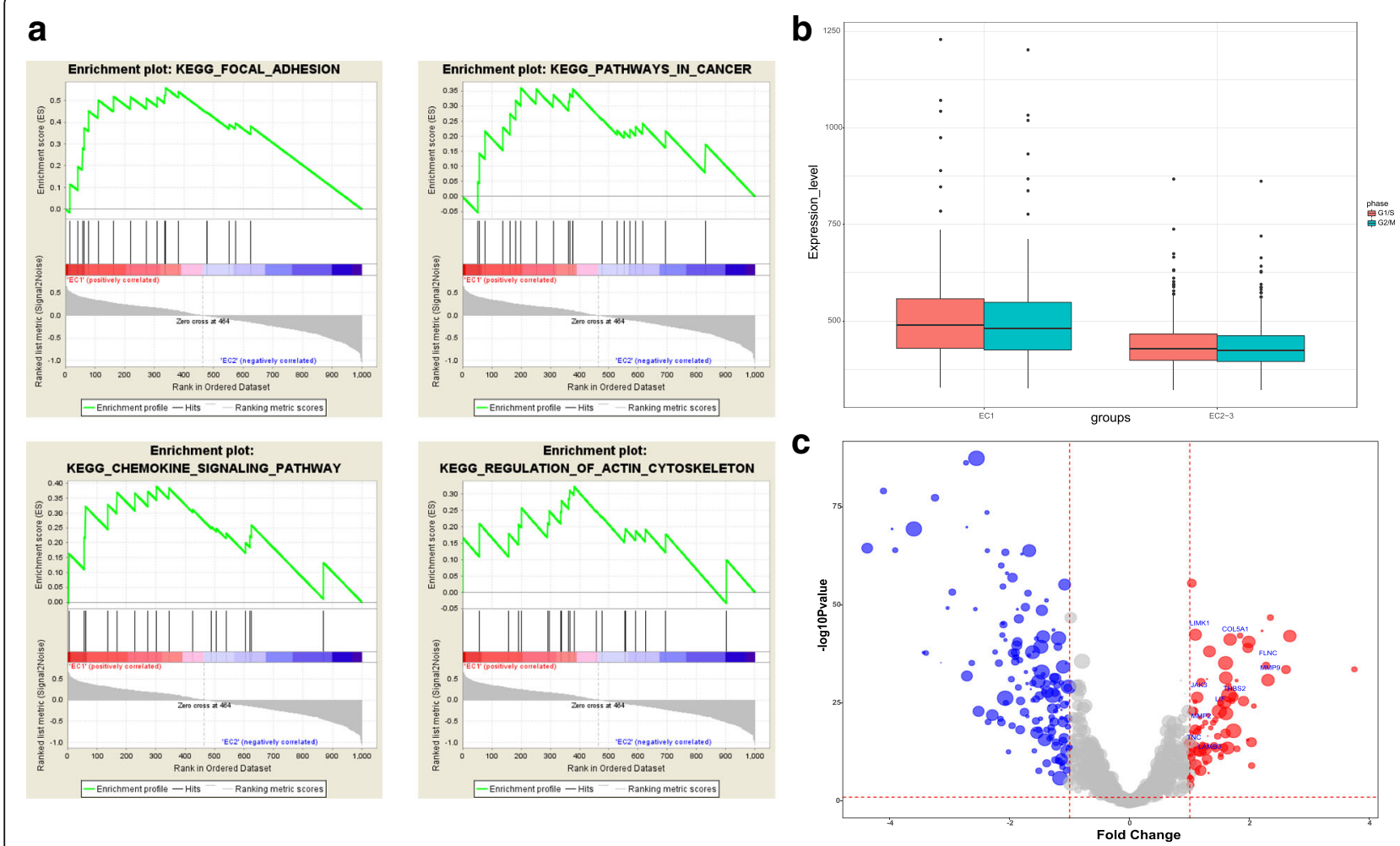

Fig. 3 a Enrichment plot of upregulation pathways in EC1. GSEA of expression data from EC1 441 with worse prognosis, as compared to EC2-3. $X$-axis is the enrichment score of each gene. Y-axis represents the order of the gene in dataset. $\mathbf{b}$ Volcano plot of differential genes. Red color: up-regulated in EC1. blue color: down-regulated in EC1. Grey: not differential genes. Size of the bubble: mean expression of each gene C box plot of mean expression level on G1/S and G2/M gene set. EC1 is higher than EC2-3. c The heatmap of ccRCC expression data of GEO. The ccRCC expression profile of GEO has similar pattern with TCGA 
are differential genes $(\operatorname{Padj}<0.01 \& \mid \log 2$ FoldChange $\mid>1)$. These genes are all high expressed in EC1 subtype (Fig. 3c).

\section{Intratumoral gene interaction network in EC1 relates to cell adhesion and motion}

After obtaining the relative enrichment pathways in $\mathrm{EC} 1$, we aim to investigate the intratumoral gene interaction network. Weighted correlation network analysis (WGCNA) algorithm (see methods) was employed to detect gene interaction modules and intramodular hub genes in EC1 subtype [26]. Seven major modules are detected and the gene co-expression pattern within these modules was very high (Additional file 7: Figure S2, Additional file 8: Figure S3). After modules were detected, we performed enrich analysis on the Seven modules we identified and blue colored module is markedly enriched with genes in pathways implicating Cell adhesion molecules, Cytokine-cytokine receptor interaction, Regulation of Actin Cytoskeleton and Chemokine Signaling Pathway (Table 4). This result, consistent with previous analysis, demonstrate that these pathways are interrelated with each other and may lead to a poor outcome for patients, which can be used as a monitoring indicator of cancer progression.

Hub genes are defined as genes that interacted with other genes most. Correlation between modules and clinical data reveals the blue module, comprised of 200 genes, demonstrating delicacy correlation with tumor stage (Additional file 9: Figure S4), may play a fatal part in the growth and metastasis of ccRCC. For the blue module, JAK3 is the hub gene filled with red color, accompanying by LIMK1 and DENND2D (Additional file 10: Figure S5), which are also hub genes but with less connectivity. JAK3 is a well-known cancer gene, playing an important role in tumorigenesis and progression of hematological malignancy [27] especially in leukemia [28, 29] and JAK3 inhibitor has been applied into treating for autoimmune and blood cancer in clinic. LIMK1, a critical regulator of actin dynamics, functioning as a regulatory role in tumor cell invasion and proliferation [30], has been reported in gastric and lung cancer [31-33]. Further studied are needed to investigate the role of JAK3 and LIMK1 in the development of ccRCC.

\section{Discrepancies in methylation levels of genes contribute to different phenotypes}

To gain insights into the methylation states of pathways and genes, we explored the methylation levels for EC1 and EC2-3 group. HM450K data of ccRCC was used and unexpectedly, higher overall methylation levels are observed in EC1 (Pvalue =3.7e-06) (Additional file 11: Figure S6). We inferred that the distinctions of methylation in specific genomic region result in the changes in expression pattern. Thus, we calculate the difference for each probe between the mean DNA methylation of each group and test for differential expression using Wilcoxon test adjusting by the Benjamini-Hochberg method to search for differentially methylated CpG sites. 93 significant hypomethylation $\mathrm{CpG}$ sites were found in EC1 subgroup (absolute beta-values difference 0.2 \& Padj < $0.01)$ and 60 are located in the protein coding region (Fig. 4a). 6 genes are related to Focal Adhesion and cell adhesion molecular in EC1 including DOCK1, LAMC1 and TLN1. We also observed epigenetic silencing of TOLLIP in EC1 and TOLLIP deficiency is associated with decreased T-cell responses [34], which may reflect the immune suppression phenomenon in EC1.

To analyze the connection between the genes that we identify involving Focal adhesion and cell adhesion molecular and prognosis of patients, we perform survival analysis and divide patients into two parts (high expression group: Zscore $>1.96$ and low expression group: Zscore $<1.96$, confidence interval $=95 \%$ ) according to

Table 4 Enrich pathways in blue module

\begin{tabular}{|c|c|c|c|c|c|}
\hline \multicolumn{6}{|l|}{ Blue Module } \\
\hline Description & Genes in Gene Set (K) & Genes in Overlap (k) & $\mathrm{k} / \mathrm{K}$ ratio & $p$-value & FDR q-value \\
\hline Cell adhesion molecules(CAMs) & 134 & 9 & 0.0672 & $1.06 \mathrm{E}-08$ & $1.97 \mathrm{E}-06$ \\
\hline Regulation of actin cytoskeleton & 216 & 10 & 0.0463 & $5.56 \mathrm{E}-08$ & $5.17 \mathrm{E}-06$ \\
\hline Chemokine signaling pathway & 190 & 9 & 0.0474 & $2.14 \mathrm{E}-07$ & $1.33 \mathrm{E}-05$ \\
\hline Cytokine-cytokine receptor interaction & 267 & 10 & 0.0375 & $3.97 \mathrm{E}-07$ & $1.82 \mathrm{E}-05$ \\
\hline Primary immunodeficiency & 35 & 5 & 0.1429 & $4.90 \mathrm{E}-07$ & $1.82 \mathrm{E}-05$ \\
\hline Leukocyte transendothelial migration & 118 & 7 & 0.0593 & $1.10 \mathrm{E}-06$ & $3.41 \mathrm{E}-05$ \\
\hline Natural killer cell mediated cytotoxicity & 137 & 7 & 0.0511 & 2.99E-06 & 7.94E-05 \\
\hline Complement and coagulation cascades & 69 & 5 & 0.0725 & $1.50 \mathrm{E}-05$ & $3.49 \mathrm{E}-04$ \\
\hline Hematopoietic cell lineage & 88 & 5 & 0.0568 & $4.88 \mathrm{E}-05$ & $1.01 \mathrm{E}-03$ \\
\hline Jak-STAT signaling pathway & 155 & 6 & 0.0387 & $7.36 \mathrm{E}-05$ & 1.37E-03 \\
\hline Toll-like receptor signaling pathway & 102 & 5 & 0.049 & $9.86 \mathrm{E}-05$ & 1.67E-03 \\
\hline
\end{tabular}


Volcano plot ( EC2-3 vs EC1)

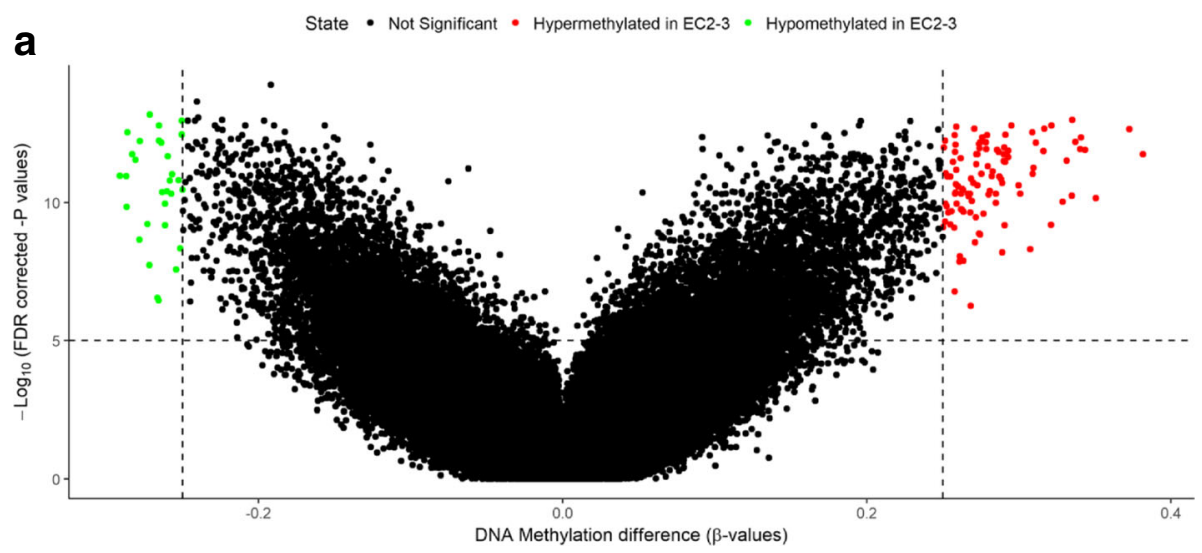

b
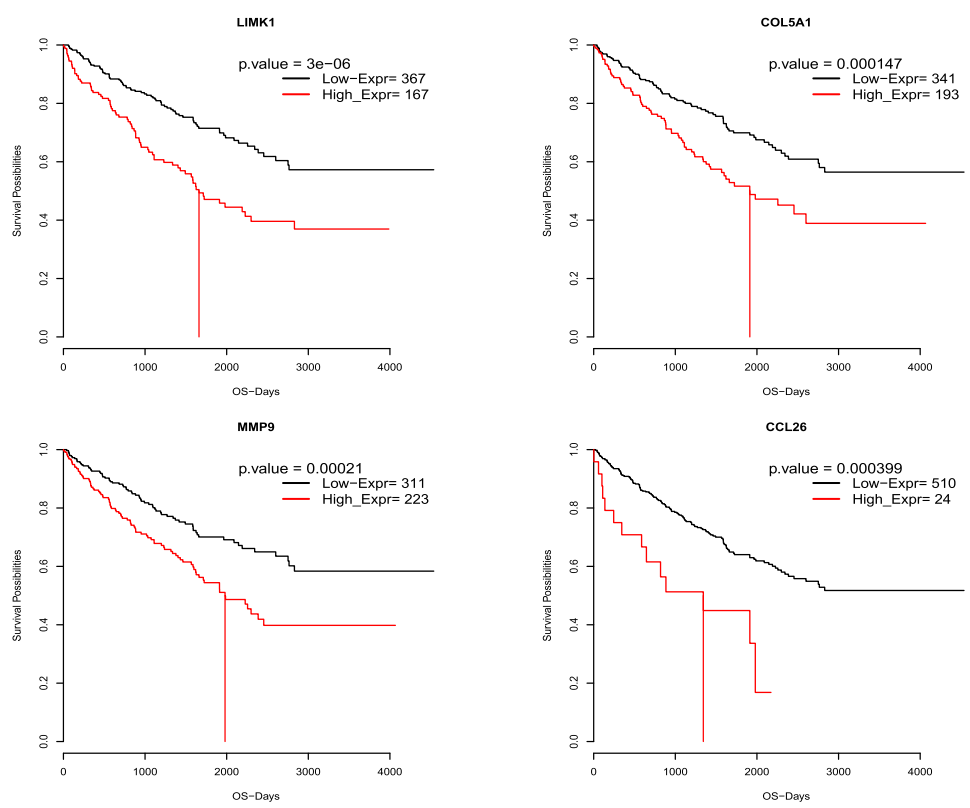

Fig. 4 a Volcano plot of differential methylation sites. Data are obtained from HM450K methylation data. $\beta$-values represent mean methylation level of CpG sites. b Kaplan meier survival plot of four genes. Red line indicates the median survival time

the expression level of genes. We find that most of the genes that enrichment or hypomethylation in EC1 are negatively related to prognosis. Four genes involving in Focal Adhesion, Pathway in cancer, Chemokine and cytoskeleton are relevant to survival evidently including LIMK1, COL5A1, MMP9 and CCL26 (Fig. 4b).

\section{Results}

We use the consensus clustering method and discover a new subpopulation of ccRCC, with a poor prognosis, higher degree of malignancy, pathological grade and clinical stage. The features of the subgroup in gene mutation, expression interation network and methylation manifest stronger potential of proliferation and metastasis, coinciding with the clinical performance which furtherly validate our findings.

\section{Discussion}

Here, we apply unsupervised Consensus Clustering algorithm and identify three distinct subtypes based on hierarchical clustering. Validation on an independent data set further illustrates the reliability of this typing. Three subtypes are characterized by divergent biological pathways and significant association with survival outcomes. In this analysis, we compare different subtypes to detect variances in pathways and also grope for the gene interaction network in the worse prognosis group. Furthermore, methylation analysis demonstrates epigenetic changes in subtypes and further validate the findings in genome and transcriptome. Our method is highly reproducible and able to identify stable categories with gene expression patterns and clinical meaning, which may be informative of tumor behavior and prognosis. 
The clinical features are markedly different in survival outcome, grade and stage between subgroups. EC1 is associated with advanced grade, stage and worse prognosis but there is no significant difference between EC2 and EC3. Given the set of characteristic subtype abnormalities, we deem it likely that patients transition between subtypes during different stages of their disease. The explanation may lie in the origin that ccRCC stem from renal tubular epithelial cells and the three subtypes present similar genetic changes including loss of $3 p$, gain of $5 q$ and somatic mutations or epigenetic alterations of VHL. Future studies on larger number of patients are needed to validate the cell origin and transition process of different subtypes.

Further analysis indicates the up-regulated pathways and hypomethylation genes mainly concentrate on Focal adhesion and mobility in EC1. Other pathways referring to Chemokine and cytokine also play as an assistant to produce progress. This kind of panel of genes in EC1 regulate EMT and cell cycle, causing tumor invasion and metastasis even before diagnosis and become aggressive and lethal compared with other subtypes. Early diagnosis and treatment are essential for patients with this class of molecular subtypes. The subtypes with better prognosis possess relatively overexpressed genes associated with hypoxia, PPAR signaling pathway and drug metabolism cytochrome P450. Intriguingly, these up-regulated genes or pathways are known to be broadly dysregulated in ccRCC. We have discovered VHL and other structural alterations in most samples across subtypes and reasoned that EC1 subtype may have acquired other genetic variations that enhance its ability of invasion and proliferation, contribute to a more aggressive phenotype and cover up the signature of VHL inactivation. In addition, it will be of interest to clarify the key changes that shape the unique subtype and elucidate the relationship between subtypes and treatment sensitivity.

\section{Conclusion}

Our cross-platform molecular analyses mirror a correlation between the EC1 subtype and worsened prognosis and highlight a number of important characteristics of genetics. Further analysis identifies some critical genes that may lead to the bad clinical outcome and become prognostic biomarkers, which will hopefully provide the foundation for the development of effective forms of therapy for this disease. Our work should lay the groundwork for an improved understanding of ccRCC molecular typing and personalized therapeutic approaches that different subtypes may require.

\section{Additional files}

Additional file 1: Figure S1. Using R package "ConsensusClusterPlus" to cluster GEO data and the cumulative distribution function (CDF) reaches a maximum when $\mathrm{k}=3$, thus consensus and cluster confidence is at a maximum. (PDF $1485 \mathrm{~kb}$ )

Additional file 2: Table S1. Enriched genes in Actin Cytoskeleton pathway. (XLSX $11 \mathrm{~kb}$ )

Additional file 3: Table S2. Enriched genes in MAPK Signal pathway. (XLSX $11 \mathrm{~kb}$ )

Additional file 4: Table S3. Enriched genes in Focal Adhesion pathway. (XLSX $11 \mathrm{~kb}$ )

Additional file 5: Table S4. Enriched genes in Pathways in Cancer. (XLSX $11 \mathrm{~kb}$ )

Additional file 6: Table S5. Enriched genes in Chemokine Signaling pathway. (XLSX $11 \mathrm{~kb}$ )

Additional file 7: Figure S2. Seven major modules of gene interaction network in EC1. (PDF $7 \mathrm{~kb}$ )

Additional file 8: Figure S3. Clustering dendrograms of genes, with dissimilarity based on topological overlap, together with assigned module colors. (PDF $30 \mathrm{~kb}$ )

Additional file 9: Figure S4. Coefficient between modules and clinical parameters. Pvalue is below coefficient value. (PDF $35 \mathrm{~kb}$ )

Additional file 10: Figure S5. Weighted Gene Co-expression Network plot of blue module. Red color means hub gene and the thickness of the line represents the connect strength of the interaction. Circle size: the number of connectivity. (PDF 607 kb)

Additional file 11: Figure S6. Boxplot of mean methylation of EC1 and EC2-3. (PDF $155 \mathrm{~kb}$ )

Additional file 12: Supplementary material and methods. (DOCX $19 \mathrm{~kb}$ )

\section{Abbreviations}

ccRCC: Clear Cell Renal Cell Carcinoma.; EC: Expression clusters.;

EMT: Epithelial-to-mesenchyme transition.; HM450K: Human Methylation

450 K Bead Chip.; SCNA: Somatic copy number analysis.; WGCNA: Weighted

Correlation Network analysis

Acknowledgments

We would like to thank all the participants that contribute to this work.

Supplementary material and methods

See the Additional file 12

Funding

Supported by Innovation Program of Shenzhen: CXZZ 20140826163906370, Development and Reform Commission of Shenzhen Municipality [2015] 1945 and Shenzhen Science and Technology Innovation Committee:

JCYJ20160429093033251 and JCYJ20140901003939019.

Availability of data and materials

All data generated or analyzed during this study are included in this published article and its supplementary information files.

\section{Authors' contributions}

Wu S., Wu P. and Liu J.L. designed the study. Wu P. and Pei S.M analyzed the patient data and carried out the genotyping. Liu J.L. and Wu C.P. performed the statistical analyzes. Wu P. wrote the manuscript. Yang K., Wang S.P., Wu C.P. contributed samples and patient information. All authors read and approved the final manuscript.

Ethics approval and consent to participate

Not applicable.

Consent for publication

Not applicable.

Competing interests

The authors declare that they have no competing interests. 


\section{Publisher's Note}

Springer Nature remains neutral with regard to jurisdictional claims in published maps and institutional affiliations.

\section{Author details}

'The Affiliated Luohu Hospital of Shenzhen University, Department of Urological Surgery, Shenzhen University, Shenzhen 518000, China. ${ }^{2}$ Shenzhen Following Precision Medical Institute, Shenzhen Luohu Hospital Group, Shenzhen 518000, China. ${ }^{3}$ Shenzhen Second People'Hospital, 1st affiliated hospital of ShenZhen University, Shenzhen 518037, China. ${ }^{4}$ College of Basic Medical Sciences, Dalian Medical University, Dalian 116044, China.

Received: 7 September 2017 Accepted: 1 March 2018 Published online: 13 March 2018

\section{References}

1. Journalist, Resource S: American Cancer Society: Cancer facts and figures 2012. Science.

2. Network CGA. Comprehensive molecular characterization of clear cell renal cell carcinoma. Nature. 2013;499(7456):43-9.

3. Gossage L, Eisen T, Maher ER. VHL, the story of a tumour suppressor gene. Nat Rev Cancer. 2015;15(1):55-64.

4. Zbar B, Brauch H, Talmadge C, Linehan M. Loss of alleles of loci on the short arm of chromosome 3 in renal cell carcinoma. Nature. 1987;327(6124): 721-4.

5. Shen C, Beroukhim R, Schumacher SE, Zhou J, Chang M, Signoretti S, Kaelin WG Jr. Genetic and functional studies implicate HIF1alpha as a 14q kidney cancer suppressor gene. Cancer Discov. 2011;1(3):222-35

6. Wilkerson MD, Hayes DN. ConsensusClusterPlus: a class discovery tool with confidence assessments and item tracking. Bioinformatics. 2010;26(12):1572-3.

7. Swift S, Tucker A, Vinciotti V, Martin N, Orengo C, Liu X, Kellam P. Consensus clustering and functional interpretation of gene-expression data. Genome Biol. 2004;5(11):R94.

8. Hayes DN, Monti S, Parmigiani G, Gilks CB, Naoki K, Bhattacharjee A, Socinsk MA, Perou C, Meyerson M. Gene expression profiling reveals reproducible human lung adenocarcinoma subtypes in multiple independent patient cohorts. J Clin Oncol. 2006:24(31):5079-90.

9. Verhaak RG, Hoadley KA, Purdom E, Wang V, Qi Y, Wilkerson MD, Miller CR Ding L, Golub T, Mesirov JP, et al. Integrated genomic analysis identifies clinically relevant subtypes of glioblastoma characterized by abnormalities in PDGFRA, IDH1, EGFR, and NF1. Cancer Cell. 2010;17(1):98-110.

10. Sturm D, Witt H, Hovestadt V, Khuong-Quang DA, Jones DT, Konermann C, Pfaff E, Tonjes M, Sill M, Bender S, et al. Hotspot mutations in H3F3A and $\mathrm{IDH} 1$ define distinct epigenetic and biological subgroups of glioblastoma. Cancer Cell. 2012;22(4):425-37.

11. Damrauer JS, Hoadley KA, Chism DD, Fan C, Tiganelli CJ, Wobker SE, Yeh JJ, Milowsky MI, lyer G, Parker JS, et al. Intrinsic subtypes of high-grade bladder cancer reflect the hallmarks of breast cancer biology. Proc Natl Acad Sci U S A. 2014;111(8):3110-5.

12. Wei X, Choudhury Y, Lim WK, Anema J, Kahnoski RJ, Lane B, Ludlow J, Takahashi M, Kanayama HO, Belldegrun A, et al. Recognizing the continuous nature of expression heterogeneity and clinical outcomes in clear cell renal cell carcinoma. Sci Rep. 2017;7(1):7342.

13. Peña-Llopis S, Vega-Rubín-De-Celis S, Liao A, Leng N, Pavía-Jiménez A Wang S, Yamasaki T, Zhrebker L, Sivanand S, Spence P. BAP1 loss defines a new class of renal cell carcinoma. Nat Genet. 2012:44(7):751-9.

14. Gu Y-F, Cohn S, Christie A, McKenzie T, Wolff NC, Do QN, Madhuranthakam A, Pedrosa I, Wang T, Dey A. Modeling renal cell carcinoma in mice: Bap1 and Pbrm1 inactivation drive tumor grade. Cancer Discovery. 2017;7(8):900.

15. Hayward NK, Wilmott JS, Waddell N, Johansson PA, Field MA, Nones K, Patch AM, Kakavand H, Alexandrov LB, Burke H, et al. Whole-genome landscapes of major melanoma subtypes. Nature. 2017;545(7653):175-80.

16. Pal SK, Ali SM, Yakirevich E, Geynisman DM, Karam JA, Elvin JA, Frampton GM, Huang X, Lin DI, Rosenzweig M, et al. Characterization of Clinical Cases of Advanced Papillary Renal Cell Carcinoma via Comprehensive Genomic Profiling. Eur Urol. 2018;73(1):71.

17. Yang $\mathrm{H}$, Kircher DA, Kim KH, Grossmann AH, VanBrocklin MW, Holmen SL, Robinson JP. Activated MEK cooperates with Cdkn2a and Pten loss to promote the development and maintenance of melanoma. Oncogene. 2017;36(27):3842-51
18. Robinson DR, Wu YM, Lonigro RJ, Vats P, Cobain E, Everett J, Cao X, Rabban E, Kumar-Sinha C, Raymond V, et al. Integrative clinical genomics of metastatic cancer. Nature. 2017;548(7667):297.

19. Mavrakis KJ, McDonald ER 3rd, Schlabach MR, Billy E, Hoffman GR, deWeck A, Ruddy DA, Venkatesan K, Yu J, McAllister G, et al. Disordered methionine metabolism in MTAP/CDKN2A-deleted cancers leads to dependence on PRMT5. Science. 2016;351(6278):1208-13.

20. Subramanian A, Tamayo P, Mootha VK, Mukherjee S, Ebert BL, Gillette MA, Paulovich A, Pomeroy SL, Golub TR, Lander ES, et al. Gene set enrichment analysis: a knowledge-based approach for interpreting genome-wide expression profiles. Proc Natl Acad Sci U S A. 2005;102(43):15545-50.

21. Qi Y, Xu F, Chen L, Li Y, Xu Z, Zhang Y, Wei W, Su N, Zhang T, Fan F, et al. Quantitative proteomics reveals FLNC as a potential progression marker for the development of hepatocellular carcinoma. Oncotarget. 2016;7(42): 68242-52.

22. Zhou Y, Dang J, Chang KY, Yau E, Aza-Blanc P, Moscat J, Rana TM. miR-1298 inhibits mutant KRAS-driven tumor growth by repressing FAK and LAMB3. Cancer Res. 2016;76(19):5777-87.

23. Tanis T, Cincin ZB, Gokcen-Rohlig B, Bireller ES, Ulusan M, Tanyel CR, Cakmakoglu B. The role of components of the extracellular matrix and inflammation on oral squamous cell carcinoma metastasis. Arch Oral Biol. 2014:59(11):1155-63.

24. Berrondo C, Flax J, Kucherov V, Siebert A, Osinski T, Rosenberg A, Fucile C, Richheimer S, Beckham CJ. Expression of the long non-coding RNA HOTAIR correlates with disease progression in bladder cancer and is contained in bladder cancer patient urinary exosomes. PLoS One. 2016;11(1):e0147236.

25. Jiang W, Crossman DK, Mitchell EH, Sohn P, Crowley MR, Serra R. WNT5A inhibits metastasis and alters splicing of Cd44 in breast cancer cells. PLoS One. 2013:8(3):e58329.

26. Langfelder $\mathrm{P}$, Horvath S. WGCNA: an R package for weighted correlation network analysis. BMC Bioinformatics. 2008;9:559.

27. Springuel L, Hornakova T, Losdyck E, Lambert F, Leroy E, Constantinescu SN Flex E, Tartaglia M, Knoops L, Renauld JC. Cooperating JAK1 and JAK3 mutants increase resistance to JAK inhibitors. Blood. 2014;124(26):3924-31.

28. Agarwal A, MacKenzie RJ, Eide CA, Davare MA, Watanabe-Smith K, Tognon CE, Mongoue-Tchokote S, Park B, Braziel RM, Tyner JW, et al. Functional RNAi screen targeting cytokine and growth factor receptors reveals oncorequisite role for interleukin-2 gamma receptor in JAK3-mutationpositive leukemia. Oncogene. 2015;34(23):2991-9.

29. Ross JA, Spadaro M, Rosado DC, Cavallo F, Kirken RA, Pericle F. Inhibition of JAK3 with a novel, selective and orally active small molecule induces therapeutic response in T-cell malignancies. Leukemia. 2014;28(4):941-4.

30. Yoshioka K, Foletta V, Bernard O, Itoh K. A role for LIM kinase in cancer invasion. Proc Natl Acad Sci U S A. 2003:100(12):7247-52.

31. Su B, Su J, Zeng Y, Liu F, Xia H, Ma YH, Zhou ZG, Zhang S, Yang BM, Wu YH, et al. Diallyl disulfide suppresses epithelial-mesenchymal transition, invasion and proliferation by downregulation of LIMK1 in gastric cancer. Oncotarget. 2016:7(9):10498-512.

32. Tan Y, Hu H, Tan W, Jin L, Liu J, Zhou H. MicroRNA-138 inhibits migration and invasion of non-small cell lung cancer cells by targeting LIMK1. Mol Med Rep. 2016;14(5):4422-8.

33. Yang X, Yu K, Hao Y, Li DM, Stewart R, Insogna KL, Xu T. LATS1 tumour suppressor affects cytokinesis by inhibiting LIMK1. Nat Cell Biol. 2004;6(7): 609-17.

34. Wells CA, Chalk AM, Forrest A, Taylor D, Waddell N, Schroder K, Himes SR, Faulkner G, Lo S, Kasukawa T, et al. Alternate transcription of the toll-like receptor signaling cascade. Genome Biol. 2006;7(2):R10. 